\title{
Model-Based Tools for Pharmaceutical Manufacturing Processes
}

\author{
René Schenkendorf ${ }^{1,2, *(\mathbb{D}}$, Dimitrios I. Gerogiorgis ${ }^{3} \mathbb{D}$, Seyed Soheil Mansouri ${ }^{4} \mathbb{D}$ and \\ Krist V. Gernaey ${ }^{4}$ (i) \\ 1 Institute of Energy and Process Systems Engineering, Technische Universität Braunschweig, \\ Franz-Liszt-Straße 35, 38106 Braunschweig, Germany \\ 2 Center of Pharmaceutical Engineering (PVZ), Technische Universität Braunschweig, Franz-Liszt-Straße 35a, \\ 38106 Braunschweig, Germany \\ 3 Institute for Materials and Processes (IMP), School of Engineering, University of Edinburgh, \\ The King's Buildings, Edinburgh EH9 3FB, UK; d.gerogiorgis@ed.ac.uk \\ 4 Department of Chemical and Biochemical Engineering, Technical University of Denmark, Building 229, \\ 2800 Kongens Lyngby, Denmark; seso@kt.dtu.dk (S.S.M.); kvg@kt.dtu.dk (K.V.G.) \\ * Correspondence: r.schenkendorf@tu-braunschweig.de
}

Received: 5 November 2019; Accepted: 27 December 2019; Published: 1 January 2020

Active pharmaceutical ingredients (APIs) are highly valuable, highly sensitive products resulting from production processes with strict quality control specifications and regulations that are required for the safety of patients. To ensure a profitable and growing pharmaceutical industry of significant societal benefits and low environmental footprint, model-based tools are fundamental to advancing the basic understanding, design, and optimization of pharmaceutical manufacturing processes in accordance with the United Nations "2030 sustainable development goals". Process analysis principles, for instance, provide a better understanding of underlying pharmaceutical manufacturing mechanisms. Model-based process design concepts facilitate the identification of optimal production and purification pathways and configurations. Process monitoring and control strategies ensure low life-cycle costs and provide new insights into critical failure modes and drug quality control issues.

The foregoing model-based concepts, and combinations of them, are key to exploring the full potential of innovative, highly effective pharmaceutical manufacturing processes. These are some of the grand challenges that can be tackled by process systems engineering (PSE), and they have been catalyzed by an unprecedented advent of established methodologies and algorithmic tools that are either available via open access environments or incorporated in commercial software/databases for a plethora of purposes (thermodynamic and solubility modeling, fluid phase equilibria, complex mixture thermophysical/mechanical property estimation, plant-wide simulation, optimization and cost estimation). The respective advances achieved using such a diversity of enabling computational technologies exemplify the Quality-by-Design $(\mathrm{QbD})$ vision and its translation into tangible artefacts and policies, illustrating how academia and industry respond to contemporary challenges for high-quality, more affordable healthcare.

This Special Issue on "Model-Based Tools for Pharmaceutical Manufacturing Processes" intends to curate novel advances in the development and application of model-based tools to address ever-present challenges of the traditional pharmaceutical manufacturing practice as well as new trends. As summarized below, the Special Issue provides a collection of nine papers on original advances in the model-based process unit, system-level, $\mathrm{QbD}$ under uncertainty, and decision-making applications of pharmaceutical manufacturing processes. The Special Issue is available online at https://www.mdpi.com/journal/processes/special_issues/pharmaceutical_processes. 


\section{Process Unit Studies}

Before complex pharmaceutical manufacturing processes can be simulated holistically, dedicated unit operations have to be studied first, including upstream and downstream processes. Starting with the synthesis of APIs and relevant intermediates, enzymatic syntheses are of particular interest as a greener, more economical, and efficient viable alternative to chemocatalytic processes. For instance, pyrimidine-nucleoside phosphorylases are highly versatile enzymes used for the production of pharmaceutically relevant intermediates. In "Dynamic Modelling of Phosphorolytic Cleavage Catalyzed by Pyrimidine-Nucleoside Phosphorylase" [1], the conversion of deoxythymidine and phosphate to deoxyribose-1-phosphate and thymine by a thermophilic pyrimidine-nucleoside phosphorylase from Geobacillus thermoglucosidasius was modeled in detail and validated experimentally including UV/Vis spectroscopy data. The resulting dynamic model might be used to identify optimal operating conditions of the enzymatic synthesis process, and can be extended to multi-enzyme reactions too.

Moreover, antibiotics are an essential group of biologics, and thus of interest in pharmaceutical manufacturing. In "Dynamic Modelling and Optimisation of the Batch Enzymatic Synthesis of Amoxicillin" [2], the batch enzymatic synthesis of the antibiotic amoxicillin, listed as a World Health Organization (WHO) "Essential Medicine", was modeled and optimized. While including non-isothermal kinetics, the authors identified an optimal temperature profile that ensures high product quality at minimum feedstock consumption.

In addition to synthesis problems, modeling of downstream processes has attracted much interest in the last few decades. For instance, spray drying is a basic unit operation in pharmaceutical manufacturing. In "Global Sensitivity Analysis of a Spray Drying Process" [3], a sensitivity analysis study of a spray drying process is discussed. To quantify the impact of different but interacting process parameters, a model-based global sensitivity analysis with a low computational cost was implemented, contributing to $\mathrm{QbD}$ and the identification of critical process parameters. These essential parameters of the process might be relevant for the development of future control strategies that can result in significant robustness for the spray drying process.

\section{System-Level Studies}

Next, based on determined process unit models, system-level studies are crucial for a detailed understanding of pharmaceutical manufacturing processes. The interaction between process units, the identification of critical process parameters, and their impact on critical quality attributes of pharmaceutical products are of key interest at the system level. For instance, when modeling the flow of material in a continuous process of several unit operations (e.g., blending, granulation, and tableting), the study of residence time distributions is the tool of choice. In "Explicit Residence Time Distribution of a Generalised Cascade of Continuous Stirred Tank Reactors for a Description of Short Recirculation Time (Bypassing)" [4], the so-called tanks-in-series model was generalized to a cascade of $n$ continuous stirred tank reactors with non-integer non-negative $n$. Therefore, the model can describe short recirculation times (bypassing) without the need for complex reactor networks. When part of a reactor network, the proposed model can be used to predict the response to upstream setpoint changes and process fluctuations, i.e., providing insights at the system level.

The relevance of model-based studies of process-wide manufacturing lines is highlighted in "Dynamic Flowsheet Model Development and Sensitivity Analysis of a Continuous Pharmaceutical Tablet Manufacturing Process Using the Wet Granulation Route" [5]. In this study, the authors implemented a dynamic flowsheet model of the ConsiGma ${ }^{\mathrm{TM}}-25$ line for continuous tablet manufacturing, including determined models of various unit operations, i.e., feeders, blenders, a twin-screw wet granulator, a fluidized bed dryer, a mill, and a tablet press. Based on the developed dynamic flowsheet model, the liquid feed rate to the granulator, the air temperature, and the drying time in the dryer were identified via global sensitivity analysis methods as critical process parameters that affect the tablet properties most. 


\section{Studies Under Uncertainty}

$\mathrm{QbD}$, an essential paradigm in pharmaceutical manufacturing, benefits from mathematical models. Model imperfections, however, have to be considered seriously; that is, uncertainty quantification and analysis are mandatory in model-based studies. This is particularly true when making use of mathematical models to study the design space of manufacturing processes. In "An Optimization-Based Framework to Define the Probabilistic Design Space of Pharmaceutical Processes with Model Uncertainty" [6], the authors introduced two algorithms to analyze the design space under uncertainties at low computational costs. The usefulness of the proposed probabilistic design space implementations was benchmarked with pharmaceutical manufacturing problems, including the Michael addition reaction as an industrial relevant case study.

In addition to uncertain model parameters and kinetics, batch-to-batch variations cause severe difficulties in pharmaceutical manufacturing, affecting drug quality, clinical studies, and therapeutics in equal measure. The joint effect of model imperfection and batch-to-batch variation is addressed in "Robust Process Design in Pharmaceutical Manufacturing under Batch-to-Batch Variation" [7]. Considering a freeze-drying process, the authors used an efficient model-based concept to predict optimal shelf temperature and chamber pressure profiles under batch-to-batch variation.

\section{Decision-Making Studies}

In addition to process analysis and optimization in pharmaceutical manufacturing, mathematical models can support the decision-making process in identifying the best manufacturing concepts in terms of reduced capital and operating costs. For instance, the best choice between pharmaceutical manufacturing process alternatives is challenging and benefits considerably from algorithms and decision-making tools. In "Online Decision-Support Tool "TECHoice" for the Equipment Technology Choice in Sterile Filling Processes of Biopharmaceuticals" [8], the authors proposed a model-based tool to support users in choosing their preferred technology according to their input of specific drug production scenarios. The usefulness of the prototype tool was demonstrated successfully with the study of equipment technologies in the sterile filling of biopharmaceutical manufacturing processes.

Modeling and simulation are a central part of research and development activities in the pharmaceutical industry, but the evaluation of modeling and simulation return on investments is difficult to quantify in advance. In "Show Me the Money! Process Modeling in Pharma from the Investor's Point of View" [9], the authors provide an algorithmic methodology that allows for the development of detailed business studies. They discuss an easy-to-use methodology that can help an investor evaluate an investment in modeling and simulation systematically.

The present Special Issue on "Model-Based Tools for Pharmaceutical Manufacturing Processes" and several more on adjacent topics which have either appeared or will be featured in Processes (but also in journals of similar scope and mission) signify the rapidly expanding importance of this research field towards securing sophisticated healthcare solutions and improving accessibility to medication for the ever-increasing and ageing global population. Publishing the fruits of academic, industrial, and collaborative efforts to this end should serve as inspiration for new challenges to set and solutions to achieve; our fervent hope is hence that PSE contributions will remain front and center in this quest.

\section{References}

1. Giessmann, R.T.; Krausch, N.; Kaspar, F.; Cruz Bournazou, M.N.; Wagner, A.; Neubauer, P.; Gimpel, M. Dynamic Modelling of Phosphorolytic Cleavage Catalyzed by Pyrimidine-Nucleoside Phosphorylase. Processes 2019, 7, 380. [CrossRef]

2. Cuthbertson, A.B.; Rodman, A.D.; Diab, S.; Gerogiorgis, D.I. Dynamic Modelling and Optimisation of the Batch Enzymatic Synthesis of Amoxicillin. Processes 2019, 7, 318. [CrossRef]

3. Bhonsale, S.; Muñoz López, C.A.; Van Impe, J. Global Sensitivity Analysis of a Spray Drying Process. Processes 2019, 7, 562. [CrossRef] 
4. Toson, P.; Doshi, P.; Jajcevic, D. Explicit Residence Time Distribution of a Generalised Cascade of Continuous Stirred Tank Reactors for a Description of Short Recirculation Time (Bypassing). Processes 2019, 7, 615. [CrossRef]

5. Metta, N.; Ghijs, M.; Schäfer, E.; Kumar, A.; Cappuyns, P.; Assche, I.V.; Singh, R.; Ramachandran, R.; De Beer, T.; Ierapetritou, M.; et al. Dynamic Flowsheet Model Development and Sensitivity Analysis of a Continuous Pharmaceutical Tablet Manufacturing Process Using the Wet Granulation Route. Processes 2019, 7, 234. [CrossRef]

6. Laky, D.; Xu, S.; Rodriguez, J.; Vaidyaraman, S.; García Muñoz, S.; Laird, C. An Optimization-Based Framework to Define the Probabilistic Design Space of Pharmaceutical Processes with Model Uncertainty. Processes 2019, 7, 96. [CrossRef]

7. Xie, X.; Schenkendorf, R. Robust Process Design in Pharmaceutical Manufacturing under Batch-to-Batch Variation. Processes 2019, 7, 509. [CrossRef]

8. Shirahata, H.; Badr, S.; Shinno, Y.; Hagimori, S.; Sugiyama, H. Online Decision-Support Tool "TECHoice" for the Equipment Technology Choice in Sterile Filling Processes of Biopharmaceuticals. Processes 2019, 7, 448. [CrossRef]

9. Varsakelis, C.; Dessoy, S.; von Stosch, M.; Pysik, A. Show Me the Money! Process Modeling in Pharma from the Investor's Point of View. Processes 2019, 7, 596. [CrossRef]

(C) 2020 by the authors. Licensee MDPI, Basel, Switzerland. This article is an open access article distributed under the terms and conditions of the Creative Commons Attribution (CC BY) license (http://creativecommons.org/licenses/by/4.0/). 